\title{
TWO DIMENSIONAL LATTICE VIBRATIONS FROM DIRECT PRODUCT REPRESENTATIONS OF SYMMETRY GROUPS
}

\author{
J.N. BOYD and P.N. RAYCHOWDHURY \\ Department of Mathematical Sciences \\ Virginia Commonwealth University \\ Richmond, Virginia 23284 U.S.A. \\ (Received October 6, 1981)
}

ABSTRACT. Arrangements of point masses and ideal harmonic springs are used to model two dimensional crystals. First, the Born cyclic condition is applied to a double chain composed of coupled linear lattices to obtain a cylindrical arrangement. Then the quadratic Lagrangian function for the system is written in matrix notation. The Lagrangian is diagonalized to yield the natural frequencies of the system. The transformation to achieve the diagonalization was obtained from group theorectic considerations. Next, the techniques developed for the double chain are applied to a square lattice. The square lattice is transformed into the toroidal Ising model. The direct product nature of the symmetry group of the torus reveals the transformation to diagonalize the Lagrangian for the Ising model, and the natural frequencies for the principal directions in the model are obtained in closed form.

KEY WOROS AND PHRASES. Group representations, direct product, Lagrangian mechanics, Born cyclic condition, Symmetry coordinates, Projection operators. 1980 MATHEMATICS SUBJECT CLASSIFICATION CODES. 20C35, $70 J 10$.

1. INTRODUCTION.

It is our purpose to extend our previous work on the applications of group representation theory in classical mechanics $[1,2,3]$. In the references cited, we have obtained the natural frequencies of vibration for monatomic and diatomic one dimensional lattices of point masses and ideal springs and have given a complete solution for natural frequencies in a one dimensional lattice in which nearest neighbors are coupled through velocities as well as position coordinates.

In brief, our solutions were obtained by replacing a one dimensional, linear array of $(\mathrm{N}+1)$ particles with a symmetric, circular arrangement of $\mathrm{N}$ particles through imposition of the Born cyclic condition. The Lagrangian function for the new model was then expressed in matrix notation. From the irreducible matrix representations 
of the symmetry group for our mechanical system, we wrote a unitary transformation matrix, $U$, with which to diagonalize the Lagrangian via a similarity transformation.

The full symmetry group for the circular model is the nonabelian, dihedral group of order $2 \mathrm{~N}$. However, by choosing the cyclic rotation subgroup of the dihedral group for computational purposes, all irreducible representations were found to be one dimensional which would not have been the case had the nonabelian, dihedral group been employed in our calculations. The one dimensional nature of the irreducible representations made the task of constructing $U$ much easier than would otherwise have been the case.

In this paper, we investigate the vibrations of a two dimensional, rectangular lattice (or Ising model) of point masses and interconnecting, ideal springs. Topologically, the two dimensional lattice may be taken as the direct product of two linear lattices, each of the sort just considered. Successive applications of the Born cyclic condition will first replace the plane lattice with a cylindrical array and then transform the cylinder into an arrangement of point masses and springs on the surface of a torus $[4,5,6]$. In turn, the torus may be regarded as the direct product of two circular arrays derived from two linear arrays by independent applications of the Born condition.

We will take the symmetry group, G, of the torus to be the direct product of the rotation groups of the factor circles in the direct product representation of the torus. It is easily seen that the operations in $G$ are symmetry elements of the torus and that $\mathrm{G}$ is abelian. However, if the original lattice is square, $\mathrm{G}$ will not be cyclic.

Since $G$ is abelian, all irreducible, matrix representations of $G$ will be one dimensional. That is, each representation is a multiplicative group of complex numbers. Since all of the irreducible representations of the abelian direct product of two finite, abelian groups can be found from the direct matrix products of the irreducible representations of the factor groups, the transformation matrix for the Lagrangian of the toroidal lattice will be $U \otimes U$, the matrix direct product of the transformation matrices for the original circular lattices [7].

Our computations will yield in closed form all the natural frequencies for vibrations in directions parallel to chains of nearest neighboring particles. One the transformation matrix has been found, the task presents no difficulties beyond the computational level. However, an appreciation of the direct product relationships among one and two dimensional lattices, symmetry groups, and representations leads to the discovery of the transformation matrix and is a significant result in itself. 2. THE DOUBLE CHAIN.

We first fix attention upon a $2 \times \mathrm{N}$ chain of identical point masses (m) as shown in Figure 1. By application of the Born cyclic condition, we take the first and last particles of each chain to be connected, thereby creating a cylindrical arrangement. The particles are numbered from left to right as 1 through $N$ in the first row and from 
left to right as $N+1$ through $2 \mathrm{~N}$ in the second row.

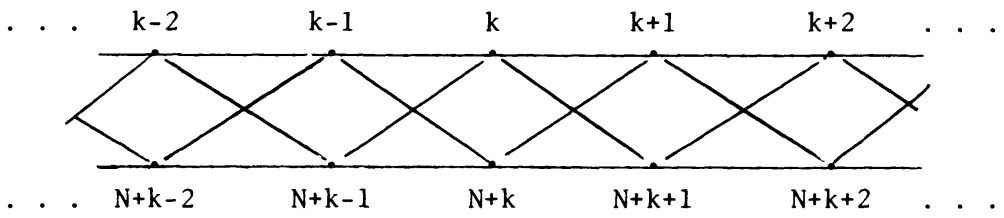

Figure 1. The Double Chain.

We allow only those motions of the system with particle displacements parallel to the length of the chain. If an only if two vertices (in Fig. 1) are connected by a line segment, the point masses at those vertices are connected by an ideal, massless spring lying along the segment. The horizontal springs have force constant $\beta_{1}$.

The diagonal springs which provide coupling between the chains and which connect next nearest neighbors have spring constant $\beta_{2}$. The diagonal springs are the source of a harmonic restoring force to oppose a shear of one chain across the other.

Vertical springs between particles $\mathrm{k}$ and $\mathrm{N}+\mathrm{k}(\mathrm{k}=1,2,3, \ldots, \mathrm{N})$ are not included. The inclusion of vertical springs would not change the elastic potential energy of the system for motions along the length of the chain since, for very small displacements of the endpoints of such vertical springs, the changes in their lengths would be negligible. Furthermore, any contribution made by such springs would be anharmonic. Thus we have a model of coupled linear lattices for which all restoring forces will be harmonic.

We denote by $x_{j}$ the displacement of the $j$-th particle from its equilibrium position along the direction parallel to the chain. The equilibrium separation of nearest neighbors along each chain is equal to the distance between chains. That is, the equilibrium positions of particles $j, j+1, N+j$, and $N+j+1$ are the vertices of a square.

$\begin{array}{ccc}j-1 & j & j+1 \\ x_{j-1} & x_{j} & x_{j+1} \\ \text { N+j-1 } & \text { N+j } & \text { N+j+1 } \\ \longrightarrow & \longrightarrow & \longrightarrow \\ x_{N+j-1} & x_{N+j} & x_{N+j+1}\end{array}$

Figure 2. Displacements from Equilibrium.

The kinetic energy of the $j$-th particle is just $\frac{1}{2} m\left(\dot{x}_{j}\right)^{2}$, and the total kinetic energy of the system can be written as $\frac{1}{2} \mathrm{~m} \dot{\mathrm{X}} \mathrm{I} \dot{\mathrm{X}}$ where $\mathrm{I}$ is the $2 \mathrm{~N} \times 2 \mathrm{~N}$ identity matrix 
and $\dot{x}$ is the column matrix of velocities $\operatorname{col}\left(\dot{x}_{1}, \dot{x}_{2}, \dot{x}_{3}, \ldots, \dot{x}_{2 N}\right)$, and $\tilde{\dot{x}}$ is the transpose of $\dot{x}$.

The elastic potential energy of the spring joining masses $j$ and $j+1$ (where $N+1$ means 1 and $2 \mathrm{~N}+1$ means $\mathrm{N}+1$ ) is

$$
\frac{1}{2} \beta_{1}\left(x_{j+1}-x_{j}\right)^{2}=\frac{1}{2} \beta_{1}\left(x_{j+1}^{2}-2 x_{j+1} x_{j}+x_{j}^{2}\right) .
$$

A simple calculation with differentials yields the result that the elastic potential energy of the spring between masses $j$ and $N+j+1(j=1,2,3, \ldots, N)$ is

$$
\frac{1}{2} \beta_{2}\left[\frac{x_{N+j+1^{-x}}}{\sqrt{2}}\right]^{2}=\frac{1}{4} \beta_{2}\left(x_{N+j+1}^{2}-2 x_{N+j+1} x_{j}+x_{j}^{2}\right) \text {. }
$$

The total elastic potential energy for the set of horizontal springs is quadratic in the displacements and may be written in the matrix form $\frac{1}{2} \beta_{1} \tilde{\mathrm{x}} V_{1} \mathrm{x}$ where $V_{1}$ is the the potential energy matrix given by $V_{1}=2 I+V_{1}^{(1)}$. The matrix $I$ is again the $2 \mathrm{~N} \times 2 \mathrm{~N}$ identity matrix and

$$
\begin{aligned}
& v_{1}^{(1)}=\left(\begin{array}{c:c}
\mathrm{v} & 0 \\
\hdashline 0 & \mathrm{v}
\end{array}\right) \text { with } \\
& \mathrm{V}=\left(\begin{array}{rrrrrrr}
0 & -1 & 0 & 0 & \ldots & 0 & -1 \\
-1 & 0 & -1 & 0 & \ldots & 0 & 0 \\
0 & -1 & 0 & -1 & \ldots & 0 & 0 \\
0 & 0 & -1 & 0 & \ldots & 0 & 0 \\
. & . & . & . & & . & . \\
. & . & . & . & & . & . \\
. & . & . & . & & . & . \\
-1 & 0 & 0 & 0 & \ldots & -1 & 0
\end{array}\right)
\end{aligned}
$$

That is, $v_{1}^{(1)}$ is the direct sum of two $N \times N$ matrices, $v_{1}^{(1)}=v \odot v$. The matrix $V$ is precisely the matrix which gives the potential energy of interaction between different particles and which appears in computations for the single, one dimensional lattice $[1,2,3]$. The displacement matrix $x=\operatorname{col}\left(x_{1}, x_{2}, x_{3}, \ldots, x_{2 N}\right)$, and $\tilde{x}$ is the transpose of $\mathrm{X}$.

Similarly, the elastic potential energy of the system of diagonal springs is given by $\frac{1}{4} \beta_{2} \tilde{\mathrm{X}} v_{2} \mathrm{X}$ where $v_{2}=2 \mathrm{I}+v_{2}^{(1)}$,

$$
v_{2}^{(1)}=\left(\begin{array}{c:c}
0 & \mathrm{v} \\
\hdashline \mathrm{v} & 0
\end{array}\right) \text {, }
$$

and $\mathrm{I}$ and $\mathrm{V}$ are as defined above.

The Lagrangian of the double chain is then

$$
L=\frac{1}{2} m \tilde{\dot{X}} I \dot{X}-\frac{1}{2} \beta_{1} \tilde{X} v_{1} X-\frac{1}{4} \beta_{2} \tilde{x} v_{2} x .
$$


Next, we must discover a suitable symmetry group for the cylindrical double chain from which to derive a transformation matrix. Reflection, $\mu$, across the center line midway between the two linear chains is a symetry operation upon the double chain and remains so after the double chain has been transformed into a cylinder and the center line into a circle.

If $E$ denotes the identity operation upon the system, we have the two-group $M=\{E, \mu\}$, a subgroup of the full symetry group of the double chain.

After application of the Born cyclic condition, the chain is also invariant under the action of the rotation group $C(N)=\left\{R, R^{2}, R^{3}, \ldots, R^{N-1}, R^{N}=E\right\}$ where $R$ is a rotation of $\frac{2 \pi}{N}$ radian about the axis of the cylinder.

The direct product

$$
C(N) \otimes M=\left\{E, R, R^{2}, \ldots, R^{N-1}, \mu, \mu R, \mu R^{2}, \ldots, \mu R^{N-1}\right\}
$$

is abelian and is also a subgroup of the full symmetry group of the double chain. Since $M, C(N)$, and $C(N) \otimes M$ are abelian, all irreducible matrix representations are one dimensional and there are $2 N$ nonequivalent representations of $C(N) \otimes M[7,8]$.

The two irreducible representations of $M$ are given by

$$
\begin{aligned}
& \Gamma_{M}^{(1)}: \Gamma_{M}^{(1)}(\mu)=-1, \Gamma_{M}^{(1)}(E)=1 ; \\
& \Gamma_{M}^{(2)}: \Gamma_{M}^{(2)}(\mu)=\Gamma_{M}^{(2)}(E)=1 .
\end{aligned}
$$

The $\mathrm{N}$ representations of $\mathrm{C}(\mathrm{N})$ are given by

$$
\Gamma_{\mathrm{C}(\mathrm{N})}^{(\mathrm{k})}: \Gamma_{\mathrm{C}(\mathrm{N})}^{(\mathrm{k})}\left(\mathrm{R}^{\ell}\right)=\exp \frac{2 \pi \mathrm{k} \ell \mathrm{i}}{\mathrm{N}} \quad \text { for } \mathrm{k}=1,2,3, \ldots \mathrm{N} .
$$

As direct products of the representations of $C(N)$ and $M$, the irreducible representations of $\mathrm{C}(\mathrm{N}) \otimes \mathrm{M}$ are

$$
\begin{gathered}
\Gamma^{(k)}: \Gamma^{(k)}\left(R^{\ell}\right)=\Gamma^{(k)}\left(\mu R^{\ell}\right)=\exp \frac{2 \pi k \ell i}{N} \\
\text { for } k=1,2,3, \ldots, N ; \\
\Gamma^{(N+k): \Gamma^{(N+k)}\left(R^{l}\right)=\exp \frac{2 \pi k \ell i}{N},} \\
\Gamma^{(N+k)}\left(\mu R^{\ell}\right)=-\exp \frac{2 \pi k \ell i}{N} \text { for } \\
k=1,2,3, \ldots, N .
\end{gathered}
$$

From these irreducible representations, we construct the $2 \mathrm{~N} \times 2 \mathrm{~N}$ unitary transformation matrix $U$ as described in the references cited $[1,2,3]$. The $4 \mathrm{~N}^{2}$ entries in $U$ are just the complex members taken from the $2 \mathrm{~N}$ irreducible representations of $\mathrm{C}(\mathrm{N}) \otimes \mathrm{M}$.

$$
U=\frac{1}{\sqrt{2 \mathrm{~N}}} U \otimes\left(\begin{array}{cc}
1 & 1 \\
1 & -1
\end{array}\right)=\frac{1}{\sqrt{2 \mathrm{~N}}}\left(\begin{array}{c:c}
\mathrm{U} & \mathrm{U} \\
\hdashline \mathrm{U} & -\mathrm{U}
\end{array}\right) \text { where }
$$

$U$ is $N \times N$ and the $(l, k)$-th entry of $U$ is $\exp \frac{2 \pi k \ell i}{N}$. That is, 


$$
\mathrm{U}=\left(\begin{array}{ccccc}
\exp \frac{2 \pi i}{\mathrm{~N}} & \exp \frac{4 \pi i}{\mathrm{~N}} & \exp \frac{6 \pi i}{\mathrm{~N}} & \ldots & 1 \\
\exp \frac{4 \pi i}{\mathrm{~N}} & \exp \frac{8 \pi i}{\mathrm{~N}} & \exp \frac{12 \pi i}{\mathrm{~N}} & \ldots & 1 \\
\exp \frac{6 \pi i}{\mathrm{~N}} & \exp \frac{12 \pi i}{\mathrm{~N}} & \exp \frac{18 \pi i}{\mathrm{~N}} & \ldots & 1 \\
\cdot & \cdot & \cdot & & \cdot \\
\cdot & \cdot & \cdot & & \cdot \\
\cdot & \cdot & \cdot & & \cdot \\
1 & 1 & 1 & \cdots & 1
\end{array}\right)
$$

Now we transform the Lagrangian given by Equation 2 .

$$
\begin{aligned}
L & =\frac{1}{2} \mathrm{~m}\left(\tilde{\dot{x}} u^{-1}\right)\left(u \mathrm{I} u^{-1}\right)(u \dot{\mathrm{x}}) \\
& -\frac{1}{2} \beta_{1}\left(\tilde{\mathrm{X}} u^{-1}\right)\left(u v_{1} u^{-1}\right)(u \mathrm{X}) \\
& -\frac{1}{4} \beta_{2}\left(\tilde{\mathrm{x}} u^{-1}\right)\left(u v_{2} u^{-1}\right)(u \mathrm{x}) \\
& =\frac{1}{2} \mathrm{~m} \tilde{\dot{N}} * I \dot{N}-\frac{1}{2} \beta_{1} \tilde{N} *\left(u v_{1} u^{-1}\right) N \\
& -\frac{1}{4} \beta_{2} \tilde{N} *\left(u v_{2} u^{-1}\right) N .
\end{aligned}
$$

The transform of $x$ is $N=\operatorname{col}\left(n_{1} n_{2} n_{3} \ldots n_{2 N}\right)$, the column matrix of symmetry coordinates; $\tilde{N}^{*}$ is the complex transpose of $N$. The symmetry coordinates are the coordinates in which the potential energy matrices will be diagonal. The complex nature of the symmetry coordinates will not affect the eigenvalues of $v_{1}$ and $v_{2}$ which determine the natural frequencies [2].

The results of the matrix computations to transform $v_{1}$ and $v_{2}$ are given below.

$$
u v_{1} u^{-1}=\left(\begin{array}{c:c}
\mathrm{U}(2 \mathrm{I}+\mathrm{V}) \mathrm{U}^{-1} & 0 \\
\hdashline 0 & \mathrm{U}(2 \mathrm{I}+\mathrm{V}) \mathrm{U}^{-1}
\end{array}\right)
$$

where $\mathrm{V}$ is given by Equation $1, \mathrm{U}$ is given by Equation 3, and

$$
\mathrm{U}(2 \mathrm{I}+\mathrm{V}) \mathrm{U}^{-1}=\left(\begin{array}{rccccc}
4 \sin ^{2} \frac{\pi}{\mathrm{N}} & 0 & 0 & \ldots & 0 \\
0 & 4 \sin ^{2} \frac{2 \pi}{\mathrm{N}} & 0 & \ldots & 0 \\
0 & 0 & 4 \sin ^{2} \frac{3 \pi}{\mathrm{N}} & \ldots & 0 \\
. & . & . & & . \\
\cdot & . & . & & . \\
. & . & . & & . \\
0 & 0 & 0 & \ldots & 0
\end{array}\right) .
$$




$$
\begin{aligned}
& U v_{2} u^{-1}=U\left(2 \mathrm{I}+v_{2}^{(1)}\right) U^{-1} \\
& =\left(\begin{array}{ccccccccccc}
2-2 \cos \frac{2 \pi}{\mathrm{N}} & 0 & 0 & \ldots & 0 & 0 & 0 & 0 & \ldots & 0 \\
0 & 2-2 & \cos \frac{4 \pi}{\mathrm{N}} & 0 & \ldots & 0 & 0 & 0 & 0 & \ldots & 0 \\
0 & 0 & 2-2 \cos \frac{6 \pi}{\mathrm{N}} & \ldots & 0 & 0 & 0 & 0 & \ldots & 0 \\
\vdots & \vdots & \vdots & & \vdots & \vdots & \vdots & \vdots & & \vdots \\
0 & 0 & 0 & \ldots & 0 & 0 & 0 & 0 & \ldots & 0 \\
0 & 0 & 0 & \ldots & 0 & 2+2 & \cos \frac{2 \pi}{\mathrm{N}} & 0 & 0 & \ldots & 0 \\
0 & 0 & 0 & \ldots & 0 & 0 & 2+2 & \cos \frac{4 \pi}{\mathrm{N}} & 0 & \ldots & 0 \\
0 & 0 & 0 & \ldots & 0 & 0 & 0 & 2+2 & \cos \frac{6 \pi}{\mathrm{N}} & \ldots & 0 \\
\vdots & \vdots & \vdots & & \vdots & \vdots & \vdots & \vdots & & \vdots \\
0 & 0 & 0 & \ldots & 0 & 0 & 0 & 0 & \ldots & 0
\end{array}\right)
\end{aligned}
$$

The equations of motion for the double chain are then given by

$$
\begin{gathered}
\frac{\mathrm{d}}{\mathrm{dt}} \frac{\partial \mathrm{L}}{\partial \dot{\eta}_{\mathrm{k}}^{*}}-\frac{\partial \mathrm{L}}{\partial \eta_{\mathrm{k}}^{*}}=0 . \\
\text { For } \mathrm{k}=1,2,3, \ldots, \mathrm{N}, \\
\ddot{m}_{\mathrm{k}}=-\left[4 \beta_{1} \sin ^{2} \frac{\pi \mathrm{k}}{\mathrm{N}}+\frac{1}{2} \beta_{2}\left(2-2 \cos \frac{2 \pi \mathrm{k}}{\mathrm{N}}\right)\right] n_{\mathrm{k}} \\
=-\left[4 \beta_{1} \sin ^{2} \frac{\pi \mathrm{k}}{\mathrm{N}}+2 \beta_{2} \sin ^{2} \frac{\pi \mathrm{k}}{\mathrm{N}}\right] \eta_{\mathrm{k}},
\end{gathered}
$$

implying natural frequencies of

$$
f_{k}=\frac{1}{2 \pi} \sqrt{\frac{4 \beta_{1} \sin ^{2} \frac{\pi k}{N}+2 \beta_{2} \sin ^{2} \frac{\pi k}{N}}{m}} .
$$

For $k=N+\ell, \ell=1,2,3, \ldots, N$,

$$
\begin{aligned}
m \ddot{\eta}_{\mathrm{N}+\ell} & =-\left[4 \beta_{1} \sin ^{2} \frac{\pi \ell}{\mathrm{N}}+\frac{1}{2} \beta_{2}\left(2+2 \cos \frac{2 \pi \ell}{\mathrm{N}}\right)\right] \eta_{\mathrm{N}+\ell} \\
& =-\left[4 \beta_{1} \sin ^{2} \frac{\pi \ell}{\mathrm{N}}+2 \beta_{2} \cos ^{2} \frac{\pi \ell}{\mathrm{N}}\right] \eta_{\mathrm{N}+\ell},
\end{aligned}
$$

implying natural frequencies of

$$
f_{N+l}=\frac{1}{2 \pi} \sqrt{\frac{4 \beta_{1} \sin ^{2} \frac{\pi l}{N}+2 B_{2} \cos ^{2} \frac{\pi l}{N}}{m}} .
$$

Thus the natural frequencies of the $2 \mathrm{~N}$ normal modes of longitudinal vibration in our model of a $2 \times \mathrm{N}$ chain have been found. 
3. THE $\mathrm{N} \times \mathrm{N}$ TWO DIMENSIONAL ISING MODEL.

We now consider the $\mathrm{N} \times \mathrm{N}$, two dimensional crystal lattice of ideal springs and point masses. The unit cell for this lattice is a simple square. We visualize this two dimensional lattice as constructed of parallel chains connected by diagonal springs just as were the chains in the previous section. By two applications of the Born cyclic condition, we take the lattice to lie on the surface of a torus. Each circular lattice is now coupled to circles on either side whereas in the double chain each chain was coupled to the other chain only.

The point masses are counted across the horizontal chains - from 1 through $\mathrm{N}$ for the first chain, from $N+1$ through $2 N$ for the second chain, ..., from $N(N-1)+1$ through $\mathrm{N}^{2}$ for the $\mathrm{N}$-th chain. Figure 3 shows the two dimensional lattice in the vicinity of the $(\mathrm{kN}+\mathrm{j})-\mathrm{th}$ point mass which is the $\mathrm{j}-\mathrm{th}$ mass in the $\mathrm{k}-\mathrm{th}$ chain. The motions are taken parallel to the chains as the arrows indicate.

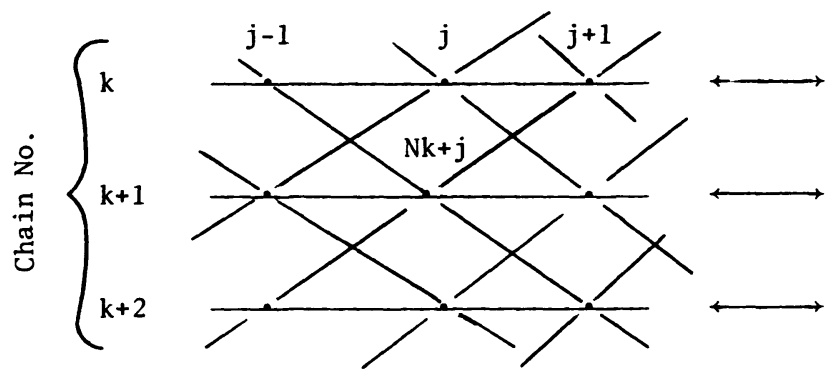

Figure 3 .

Again the horizontal springs, which are parallel to the motions of the system, have force constant $\beta_{1}$, and the diagonal springs between next nearest neighbors have force constant $\beta_{2}$.

The potential energy matrices will be $\mathrm{N}^{2} \times \mathrm{N}^{2}$ and can be constructed from the $\mathrm{N} \times \mathrm{N}$ matrix $\mathrm{V}$ defined by Equation 1 . The potential energy for the horizontal springs is quadratic in the displacements $\left.x_{1}, x_{2}, x_{3}, \ldots x_{(N}{ }^{2}\right)$. By the same reasoning which led to $V_{1}$ for the double chain we have $V_{1}=2 I+V(1)$ where $I$ is the $N^{2} \times N^{2}$ identity matris and

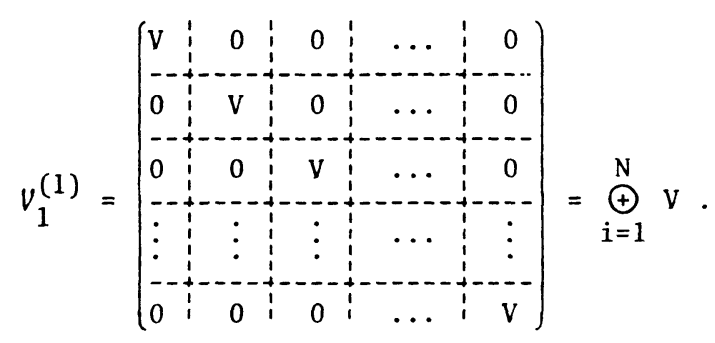


Then the potential energy for the horizontal springs is $\frac{1}{2} \beta_{1} \tilde{x} V_{1} x$, and $x$ is the column matrix $x=\operatorname{col}\left(x_{1} x_{2} x_{3} \cdots x_{(N 2)}\right)$.

The potential energy matrix, $V_{2}$, for the diagonal springs requires the inclusion of the $\mathrm{N} \times \mathrm{N}$ matrix $\mathrm{V}$ twice in each row of the block form since each horizontal chain is coupled to two other chains. For the same reason, 4 I replaces $2 \mathrm{I}$ which appeared when the matrix for the double chain was written.

For the diagonal springs of the $\mathrm{N} \times \mathrm{N}$ Ising model, we have $v_{2}=4 \mathrm{I}+v_{2}^{(1)}$ where

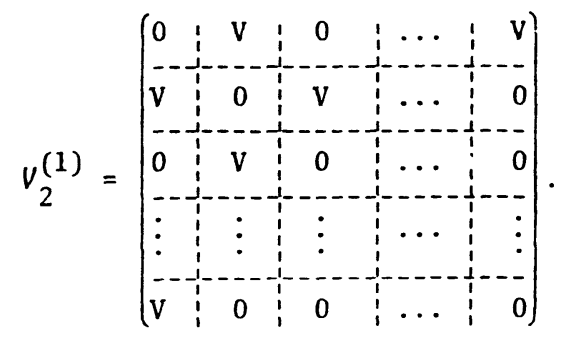

Note that the block form of $v_{2}^{(1)}$ is exactly the same as that of $V$ when $v$ in $v_{2}^{(1)}$ corresponds to -1 in $\mathrm{V}$.

The potential energy contribution by the diagonal springs is $\frac{1}{4} \beta_{2} \tilde{x} V_{2} x$.

The kinetic energy is simply $\frac{1}{2} m \tilde{x} \dot{X} \dot{X}$, and the Lagrangian becomes

$$
L=\frac{1}{2} m \stackrel{\gamma}{x} I \dot{X}-\frac{1}{2} \beta_{1} \tilde{X} v_{1} X-\frac{1}{4} \beta_{2} \tilde{x} V_{2} x
$$

We take as the symmetry group of the system the direct product group $C(N) \subseteq C(N)$. In the case of the double chain, the reflection group $M$ is isomorphic to $C(2)$, the rotation group of order 2 . In general, if the lattice were $N \otimes L$, we would take the symmetry group $\mathrm{C}(\mathrm{N}) \otimes \mathrm{C}(\mathrm{L})$. The construction of the unitary tranformation matrix $U$ is analogous to that for the double chain. In this case,

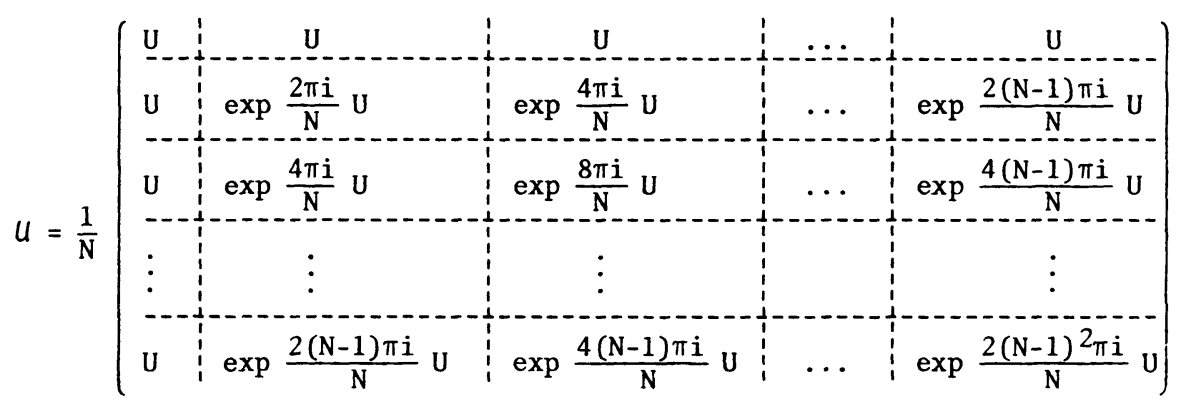

where $U$ is given by Equation 3 .

The matrix multiplications are straightforward but tedious as we diagonalize the Lagrangian. The tricks involved are just those which are useful in the one dimensional case $[1,2,3]$. We employ the same notation for the symmetry coordinates as we did in the 
case of the double chain and write $N=U x$ with $N=\operatorname{col}\left(n_{1} n_{2} n_{3} \ldots n_{(N 2)}\right)$.

The transformed Lagrangian is

$$
\begin{aligned}
L=\frac{1}{2} m \tilde{\tilde{N}} * I \dot{N} & -\frac{1}{2} \beta_{1} \tilde{N} *\left(u v_{1} u^{-1}\right) N \\
& -\frac{1}{4} \beta_{2} \tilde{N} *\left(u v_{2} u^{-1}\right) N .
\end{aligned}
$$

The matrix $u v_{1} u^{-1}$ consists of $\mathrm{N}$ diagonal blocks each of which is the $\mathrm{N} \times \mathrm{N}$ matrix

$$
\left(\begin{array}{ccccc}
4 \sin ^{2} \frac{\pi}{N} & 0 & 0 & \cdots & 0 \\
0 & 4 \sin ^{2} \frac{2 \pi}{N} & 0 & \cdots & 0 \\
0 & 0 & 4 \sin ^{2} \frac{3 \pi}{N} & \cdots & 0 \\
\vdots & \vdots & \vdots & & \vdots \\
0 & 0 & 0 & \cdots & 0
\end{array}\right)
$$

The matrix $U V_{2} U^{-1}$ has the block diagonal form

$$
\left(\begin{array}{lllll}
\mathrm{A}_{1} & 0 & 0 & \cdots & 0 \\
0 & \mathrm{~A}_{2} & 0 & \cdots & 0 \\
0 & 0 & \mathrm{~A}_{3} & \cdots & 0 \\
\vdots & \vdots & \vdots & & \vdots \\
0 & 0 & 0 & \cdots & \mathrm{A}_{\mathrm{N}}
\end{array}\right)
$$

where $A_{k}$ is the $N \times N$ matrix given by

$$
A_{k}=\left(\begin{array}{ccccc}
4-4 \cos \frac{2(k-1) \pi}{N} \cos \frac{2 \pi}{N} & 0 & 0 & \cdots & 0 \\
0 & 4-4 \cos \frac{2(k-1) \pi}{N} \cos \frac{4 \pi}{N} & 0 & \cdots & 0 \\
0 & 0 & 4-4 \cos \frac{2(k-1) \pi}{N} \cos \frac{6 \pi}{N} & \ldots & 0 \\
\vdots & \vdots & \vdots & & \vdots \\
0 & 0 & 0 & \cdots-4 \cos \frac{2(k-1) \pi}{N}
\end{array}\right)
$$

for $k=1,2,3, \ldots, N$.

Finally, putting all these computations together, we have the terms in the Lagrangian which involve $n_{\ell N+k}$ and $\eta^{*}{ }_{\ell N+k}$ to be 


$$
\begin{aligned}
& \mathrm{L}\left(n_{\ell N+k}, n_{\ell N+k}^{*}, \dot{n}_{\ell N+k}, \dot{n}_{\ell N+k}^{*}\right)= \\
& \frac{1}{2} \dot{m}_{\ell N+k} \dot{\eta}_{\ell N+k}^{*}-\frac{1}{2} \beta_{1}\left(4 \sin ^{2} \frac{k \pi}{N}\right) \eta_{\ell N+k} n_{\ell N+k} \\
& -\frac{1}{4} \beta_{2}\left(4-4 \cos \frac{2 \ell \pi}{N} \cos \frac{2 k \pi}{N}\right) \eta_{\ell N+k} \ell_{\ell N}^{*}+k
\end{aligned}
$$

Then as a function the integers $k$ and $\ell, k=1,2,3, \ldots, N, \ell=0,2,3, \ldots, N-1$, the equation of motion for the $(\ell N+k)-t h$ symmetry coordinate is

$$
m \ddot{n}_{\ell N+k}=-\left(4 \beta_{1} \sin ^{2} \frac{k \pi}{N}+2 \beta_{2}-2 \beta_{2} \cos \frac{2 \ell \pi}{N} \cos \frac{2 k \pi}{N}\right) n_{\ell N+k}
$$

Therefore, for the ordered pair of integers $(\ell, k)$, we have the natural frequency

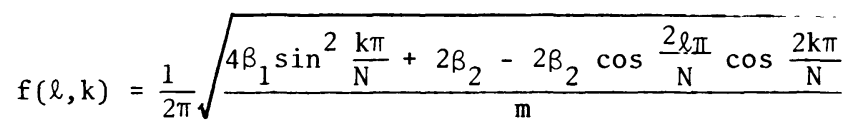

Since there are $\mathrm{N}^{2}$ such pairs of integers, we have all the different natural frequencies. Note that $(l, k)=(0, N)$ corresponds to the frequency $f(0, N)=0$ implying a translation.

If we make a change of variables to obtain a notation which is more familiar in solid state physics, we can write

$$
f\left(\ell^{\prime}, k^{\prime}\right)=\frac{1}{2 \pi} \sqrt{\frac{4 \beta_{1} \sin ^{2} \frac{k^{\prime} \pi}{N}+2 \beta_{2}-2 \beta_{2} \cos \frac{2 \ell^{\prime} \pi}{N} \cos \frac{2 k^{\prime} \pi}{N}}{m}}
$$

where $-\frac{N}{2}<\ell^{\prime} \leq \frac{N}{2},-\frac{N}{2}<k^{\prime} \leq \frac{N}{2}$. We have the maximum possible frequency for $\left(\ell^{\prime}, k^{\prime}\right)=\left(0, \frac{N}{2}\right)$. This maximum frequency is $\frac{1}{\pi} \sqrt{\frac{\beta_{1}+\overline{\beta_{2}}}{m}}$. The possible values of $\ell^{\prime}$ and $k^{\prime}$ define a square Brillouin zone in an $\left(\ell^{\prime}, k^{\prime}\right)$-space with vertices at $\left(\ell^{\prime}, k^{\prime}\right)=$ $\left( \pm \frac{N}{2}, \pm \frac{N}{2}\right)$. The zero frequency occurs at $(0,0)$.

4. CONCLUSIONS.

The simplifications of the problem of the two dimensional mechanical Ising model were made possible by appreciation of the direct product nature of the torus and its symmetry group.

We conclude with three observations about the results for the natural frequencies. First, the frequencies reduce to those of $N$ independent linear chains if $\beta_{2}$ is taken to be zero.

Next, we note that the computations for the double chain were included in this paper since it was the insight into the mathematics which we gained in doing the simpler problem that enabled us to see our way through the general problem. In any event, the double chain is interesting in itself, and the results are consistent with those for the general problem.

Finally, we can observe that if vertical springs are included, we would have the same frequencies for displacements parallel to these vertical springs since we would 
then ignore the contributions of the horizontal springs to the total potential energy. Thus we have all frequencies along the principal directions of our general $N \times N$ two dimensional lattice.

\section{REFERENCES}

1. J. N. Boyd and P. N. Raychowdhury, "An Application of Projection Operators to a One Dimensional Crystal," Bulletin of the Institute of Mathematics, Academia Sincia 7 (1979), 133-44.

2. J. N. Boyd and P. N. Raychowdhury, "Representation Theory of Finite Abelian Groups Applied to a Linear Diatomic Crystal," International Journal of Mathematics and Mathematical Sciences 3 (1980), 559-74.

3. J. N. Boyd and P. N. Raychowdhury, "A One Dimensional Crystal With Nearest Neighbors Coupled Through Their Velocities," ASME Journal of Dynamic Systems, Measurement and Control 103 (1981), 293-6.

4. J. N. Boyd and P. N. Raychowdhury, "Group Representations in Lagrangian Mechanics: An Application to a Two-Dimensional Lattice," Physica 114A (1982), 604-8.

5. Huang, K., Statistical Mechanics (John Wiley, New York, 1963).

6. Thompson, C. J., Mathematical Statistical Mechanics (Macmillan Company, New York, 1972).

7. Joshi, A. W., Elements of Group Theory for Physicists (Halsted Press, New York, 1973).

8. Hamermesh, M., Group Theory and Its Applications to Physical Problems (Addison Wesley, Reading, MA., 1962). 


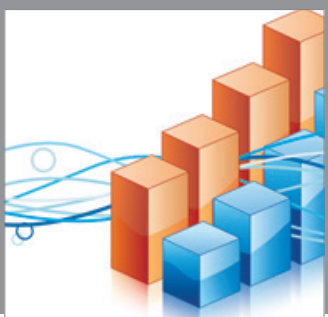

Advances in

Operations Research

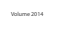

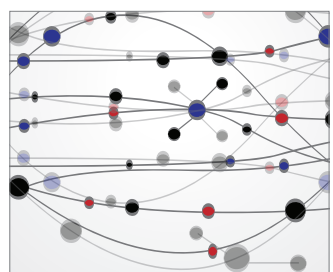

\section{The Scientific} World Journal
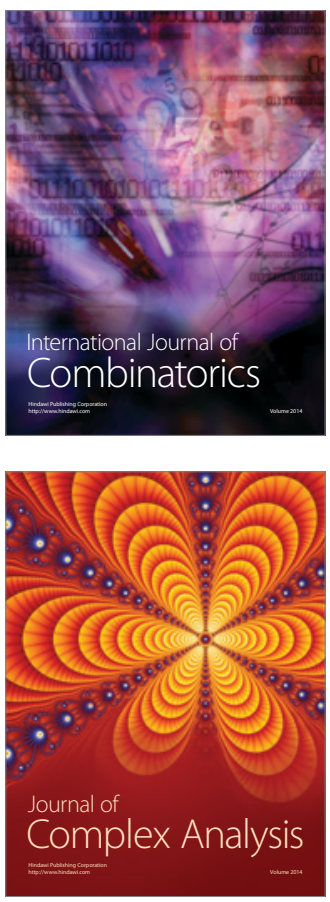

International Journal of

Mathematics and

Mathematical

Sciences
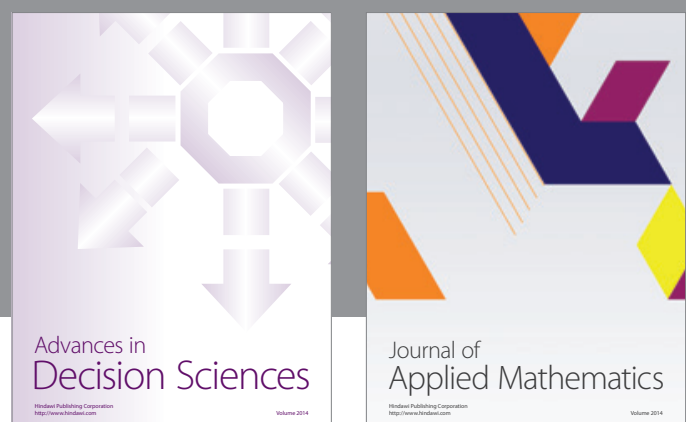

Journal of

Applied Mathematics
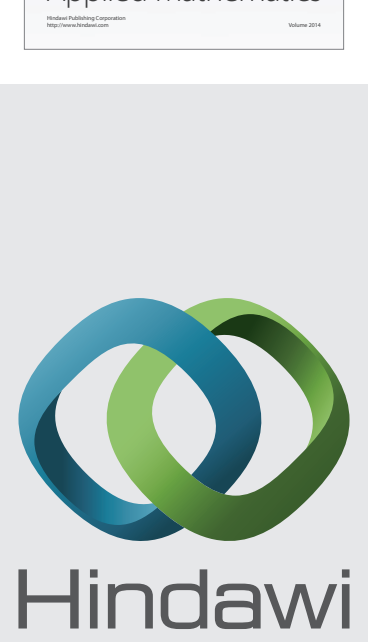

Submit your manuscripts at http://www.hindawi.com
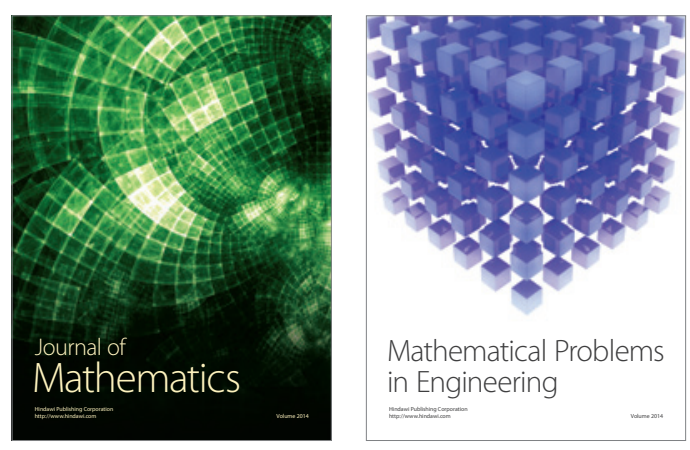

Mathematical Problems in Engineering
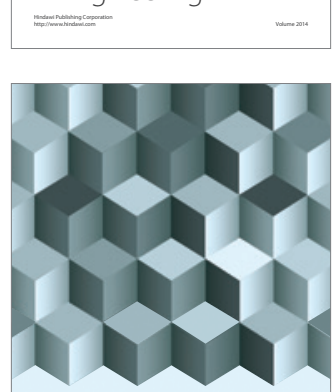

Journal of

Function Spaces
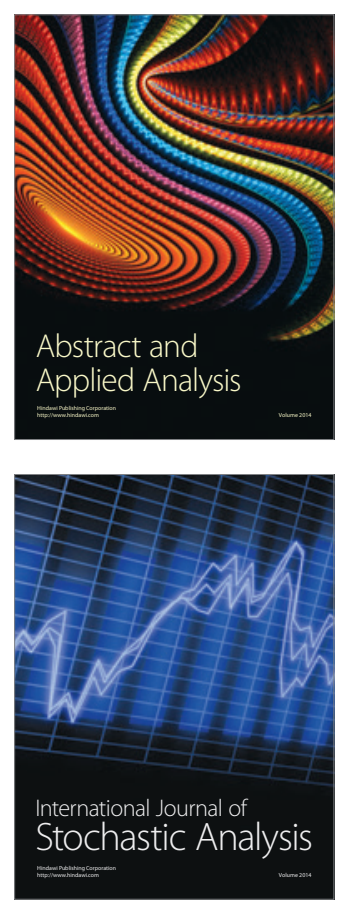

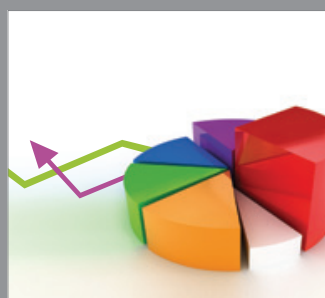

ournal of

Probability and Statistics

Promensencen
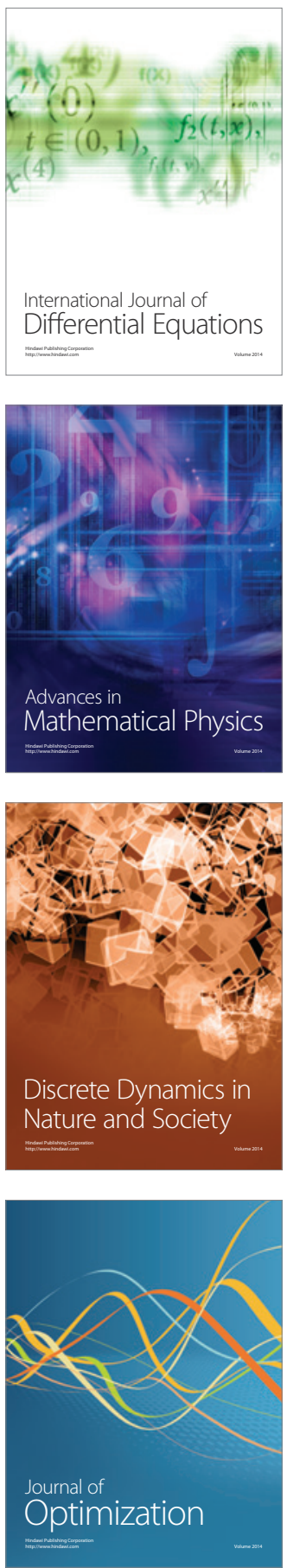